\title{
Robust Inferential Control for a Packed-Bed Reactor
}

\author{
Hector M. Budman, Christopher Webb, and Manfred Morari* \\ Chemical Engineering 210-41 \\ California Institute of Technology \\ Pasadena CA 91125
}

\begin{abstract}
An inferential robust control technique is applied to an experimental fixed bed reactor. The controlled variables are the exit concentration and the maximum bed temperature. Both controlled variables are inferred from one single temperature measurement. The location of this measurement is selected to optimize the performance of the closed-loop system when model uncertainty is allowed. Cloeed-loop experiments are conducted to test the ro bustness characteristics of the controller. From these experiments, the operating regions most sensitive to modelling uncertainty are determined. The nonlinear system characteristics can cause significant offset in the inferred controlled variables.
\end{abstract}

\section{Introduction}

The application of inferential robust control to a fixed bed methanation reactor is studied. The experimental system is described in detail by Webb et al [1]. Webb operated the reactor under conditions of full conversion. In the present work, we turn our attention to the problem of controlling the reactor under conditions of partial conversion. The controlled variables are the maximum bed temperature and the outlet concentration. The control objectives are to obtain stability and good performance over a range of different operating conditions.

In practice, it can be problematic to measure concentration in real time. First, gas chromatographs are relatively expensive to operate and maintain. In addition, gas chromatographs have relotively slow sampling rates. This slow sampling rate can affect the stability and deteriorate the performance of the closed-loop control system. For these reasons, one often infers the concentration from temperature measurements (inferential control).

There are two important issues in the design of an inferential control scheme:

-Which measurements should be selected for good closed-loop performance?

-Given a set of measurements, how does one design an inferen. tial controller which is robust to changes in the operating condi tions?

In this work a measurement selection technique derived from Structured Singular Value Theory [2] is applied to the experimental reactor problem. The optimal measurement choice depends on the selected controller. In the present work the Internal Model Control (IMC) method is used for designing the controller since it leads to relatively simple criteria for measurement selection.

\section{Inferential Control}

Let the system be described by the following dynamic model:

$$
\begin{aligned}
& s=G_{\imath d} d+G_{\imath m} m \\
& c=G_{e d} d+G_{e m} m
\end{aligned}
$$

where

s-vector of secondary measurements,

c-vector of controlled variables, m-vector of manipulated variables,

\footnotetext{
"Author to whom correpondence should be adremed mmOimc.caltech.edu, phone (818)356-4186
}

d-vector of unmeasured disturbances,

$G_{i j}$-transer function relating output vector $i$ to input vector $j$.

Inferential control [3] uses secondary variables, temperature measurements in the present study, to infer the effects of unmeasured disturbances on the controlled variables. An estimator is constructed which measures the secondary variables and predicts the effective change in the controlled variables.

If the number of independent measurements $s$ is at least equal to the number of disturbances $d$, then a perfect estimator for $c$ can be obtained from

$$
E=G_{e d} G_{e d}^{-1}
$$

when $G_{s d}$ is stably invertible.

The controller is designed to compensate for the estimated changes in the controlled variables.

A number of researchers have examined the measurement selection problem for inferential control and several different approaches have been proposed. Among others Kumar and coworkers [4] and Harris and coworkers [5] examined the problem in a stochastic state space framework. Model uncertainty is accounted for by introducing additive random disturbances .

Weber [3] proposed a steady state criterion for measurement selection assuming a specific uncertainty structure in the matrix $\mathrm{G}_{\boldsymbol{\alpha} \cdot \text {. }}$.

The measurement selection method, proposed by Lee[2], describes model mismatch as norm bounded-uncertainty of general structure on the empirical transfer functions which described the process. This general technique is summarized in the following section.

\section{Robust Inferential Control}

Using the inferential control framework, Lee and Morari [2] propose a new methodology for measurement selection and controller design based on Structured Singular Value theory. This analysis allows for structured norm-bounded uncertainty in each one of the system transfer matrices as well as frequency domain performance specifications.

In the present work, we will apply this messurement selection technique to the experimental reactor problem. Figure la shows the block diagram under consideration. In this figure,

$$
\Delta_{v}=\left[\begin{array}{ccc}
\Delta_{i}^{*} & & \\
& \ddots & \\
& & \Delta_{i}^{*}
\end{array}\right] \quad \quad \bar{\sigma}\left(\Delta_{i}^{*}\right) \leq 1
$$

where $\Delta_{i}^{*}$ are the uncertainties associated with the transfer matrices which model the process. Lee assumed the uncertainty to be cornplex and dependent on frequency. This type of uncertainty gives rise to a family of linear time invariant plants. The disturbances $d$ and the controlled variables $c$ are normalized by the frequency dependent transfer matrices $W_{d}$ and $W_{p}$, respectively. The performance weight $W$, is selected to reflect the relative importance of the errors in the controlled variables. The controller, $Q=\bar{Q} E$, is chosen so that the nominal system is cloeed-loop stable. This is true if and only if $Q$ is stable. The eloeed loop in 
Fig $1 \mathrm{a}$ is axid to achieve robust performance [6] if the closed loop system is nominally stable (i.e. stable with $\Delta_{a}=0$ ) and:

$$
\max _{\Delta=\in \Delta_{\mathbf{u}}}\left\|F_{\text {eUd }}\left(Q, \Delta_{\mathbf{u}}\right)\right\|_{\infty}<1
$$

where $F_{e^{\prime}} d$ denotes the clooed loop tranafer matrix from $d$ to $c$. Based on this condition, for each poesible set of secondary measurements, a controller can be selected to solve the following optimization problem:

$$
\phi=\min _{Q \in R H_{-}, \Delta_{v} \in \Delta_{\mathrm{u}}} \max _{\mathrm{q}} \bar{\sigma}\left[F_{\text {cid }}\left(Q, \Delta_{\mathbf{u}}\right)\right]
$$

The measurement set which minimizes over all the available ets, is the one that has to be selected for inference. Since this is a very difficult optimization problem, Lee and Morari restrict the analysis to a specific type of control, the Internal Model Control(MC). Using the IMC design procedure, $\tilde{Q}$ is split into two parts, $\tilde{Q}=\tilde{Q}, F$, where $F$ is a simple robustnese filter (usually of the form $g(s) I)$ and $\tilde{Q}_{n}$ is either an $\mathbf{H}_{2}$ or $\mathbf{H}_{\infty}$ optimal controller designed for the nominal conditions. If $G_{c o m}$ has a stable right inverse, $\bar{Q}_{n}=\left(G_{c m}\right)_{r}^{-1}$ is selected, otherwise $\bar{Q}_{n}$ is an approximate inverse of $\mathrm{G}_{\mathrm{cm}}$.

Lee uses reparate criteria for the steady state and for the general dynamic case. Since the steady state criterion requires less computational effort it can be used initially to reduce the number of candidate measurement sets. In some chemical processes with slow dynamics and stow disturbances the steady state criterion may be sufficient. However, in general the final selection has to be based also on dynamic considerations. In the sequel, both the steady state criterion and general dynamic measurement selection criterion will be summarized.

\subsection{Steady State Measurement Selection: The- ory.}

The measurement selection is based on the minimization of the morst-possible closed-loop steady state error:

$$
\max _{\alpha(0)} \frac{\left\|W_{2} c(0)\right\|_{2}}{\left\|W_{2}^{-1} d(0)\right\|_{2}} \triangleq \frac{1}{5}
$$

for the estimator and the controller presented above. The block diagram shown in Fig $1 \mathrm{a}$ can be put into the $M-\Delta$ form shown in Fig lb. The quantity $c_{p}^{*}$ in this figure is a sealar which multiplies the performance weight $W_{p}$. Considering this $M-\Delta$ structure the Robust Performance condition can be formulated in terma of $\mu$-the Structured Singular Value[6]. The value of $c ;$ can be computed from the following equation:

$$
{ }^{\mu}\left[\begin{array}{ll}
\Delta_{\mathrm{u}}^{*} & \\
& \Delta_{\mathrm{p}}
\end{array}\right]\left(\begin{array}{cc}
M_{11} & M_{12} \\
c_{p}^{*} M_{21} & c_{p}^{*} M_{22}
\end{array}\right)=1
$$

The measurement selection is based on $c^{*}$ even though this quantity is derived from a sufficient condition. The measurement set which maximizes $c_{;}^{*}$ has the smallest potential steady state error and should be the first measurement considered for control design. If $c_{p}^{*}<1$ then the worst possible clooed loop performance may exceed the performance criterion, as expressed by $W_{p}$, and the measurement set can be eliminated from further consideration. On the other hand, if $c ;>1$, robust performance at steady state is guaranteed.

\subsection{Dynamic Measurement Selection:Theory.}

In this case the criterion is based on the idea of multivariable bop shaping[6]. The cloeed loop with the proposed IMC controller is shown in Fig 2a. Since $\tilde{Q}_{n}$ in the MMC design is selected as an approximate inverse of $G_{e m}$, the filter $F$ corresponds roughly to the "complementary sensitivity". The clooed loop block diagram in Fig. $2 a$ can be put into the $\mathrm{N}-\Delta$ form shown in Fig. 2b. For this $\mathrm{N}-\Delta$ interconnection strueture it is possible to compute frequencydependent bounds on $\bar{\sigma}(F)$ and $\bar{\sigma}(I-F)\left(c_{F}\right.$ and $\left.c_{I-F}\right)$ and make the measurement selection based on the restrictiveness of these bounds. If a filter can be found such that $\bar{\sigma}(F)<c_{F}$ or $\bar{\sigma}(I-F)<$ $c_{I-F} \forall \omega$ then robust performance is guaranteed. If no filter can be found to meet these requirements, then a different messurement set should be examined. The bounds can be regarded as bounds on the sensitivity and complementary sensitivity of the closed loop. The connection between the steady state criteria and these bounds is that the condition $\frac{1}{f} \leq 1$ is a necessary condition for the existence of a nonzero $c_{I-F}$ at steady state.

\section{Application of Robust Inferential Control to the Methanation Reactor}

In this section, the robust control techniques for measurement selection and control design presented in the previous section are applied to the concentration control of an experimental fixed bed reactor. All aspects of the controller design will be covered including the description of the model uncertainty, robust measurement selection, design of the dynamic compensator, and the implementation of the controller on the reactor.

\subsection{Control Problem}

The reaction studied is the methanation of $\mathrm{CO}_{2}$ :

$$
\mathrm{CO}_{2}+4 \mathrm{H}_{2} \rightarrow \mathrm{CH}_{4}+\mathrm{H}_{2} \mathrm{O}
$$

This reaction is exothermic and irreversible under normal operating conditions. It is catalyzed by a commercial nickel catalyst with which no significant side reaction has been obeerved. The reactor consists of a single $2.5 \mathrm{~cm} \times 60 \mathrm{~cm}$ long stainless steel tube filled with a mixture of catalyst and inert alumina. Running down the center of the reactor is a $3 \mathrm{~mm}$ wide stainless steel tube which contains 15 thermocouples at different axial positions, and surrounding the reactor tube is a bath of boiling Dowtherm. This fluid maintains a constant temperature at the wall of the reactor tube. This temperature is varied by manipulating the pressure of the bath.

The flow rates of reacting gases, $\mathrm{CO}_{2}$ and $\mathrm{H}_{2}$, as well as the inert gas, $\mathrm{N}_{2}$, are individually controlled using mass flow controllers. The temperature of the resulting mixed stream is regulated using a heating tape. The concentration of the product gas is determined using a gas chromatograph. The product stream is sampled every $3.5 \mathrm{~min}$ and the results of the analysis are stored in a personal computer where they can be easily referenced off-line. A compressor connected in series to the reactor enables to recycle the products stream.

The control objective is to regulate the exit coneentration and the maximal bed temperature over a diverse set of operating conditions. These operating conditions are listed in Table 1 and include both partial and complete conversion of the reactant $\mathrm{CO}_{2}$. For the partial conversion conditions, the maximum bed temperature occurs at the reactor exit whereas for full conversion a hot spot forms inside the reactor.

\begin{tabular}{|c|c|c|}
\hline Variable & Amount & Units \\
\hline \hline Exit $\mathrm{CO}_{2}$ Concentration & $0-1.5$ & $\%$ vol \\
\hline Exit Temperature & $268-290$ & ${ }^{\circ} \mathrm{C}$ \\
\hline Inlet Heating Porrer & $0-30$ & $\%$ of 1800 capacity \\
\hline Recycle Flow rate & $10-18$ & slpm \\
\hline Inlet $\mathrm{CO}_{2}$ Concentration & 3.5 & $\%$ vol \\
\hline $\mathrm{CO}_{2}$ Feed Flow rate & 0.44 & Ilpm \\
\hline $\mathrm{H}_{2}$ Feed Flow rate & 1.96 & slpm \\
\hline $\mathrm{N}_{2}$ Feed Flow rate & 10 & dpm \\
\hline Dowtherm Temperature & $253-257$ & $\circ$ \\
\hline
\end{tabular}

Table 1:Operating Conditions for contro experiments.

The manipulated variables are the recycle fiow rate and the power supplied to the tape heaters at the inlet of the reactor. Increasing the recycle flow rate increases the space velocity in the reactor and makes the reactor behave (at least kinetically) more 
like a continuous stirred tank reactor [7]. The methanation reaction varies from first order type for low $\mathrm{CO}_{2}$ concentration to zero order for bigh concentration of this reactant. Based on this fact it can be shown [7] that the steady atate $\mathrm{CO}_{2}$ exit concentration increases and the maximum bed temperature decreases for increasing recycle flowrates. For the power supplied to the heaters, the situation is revered. Increases in the power tend to increase the maximum temperature and decrease the exit reactant concentration.

We considered disturbances in the reactor wall temperature only. Physically, this temperature is equal to the temperature of the boiling Dowtherm fluid which surrounds the reactor. There are two reasons for changes in the Dowtherm temperature:

Pressure changes inside the Dowtherm container.

The Dowtherm fluid does not boil uniformly. As a result spatial temperature variations can occur due to a sudden increase of the flow rate through the reactor.

It will be shown that the steady state bed temperatures and the exit concentration change aignificantly with small changes in the wall temperature.

\subsection{Uncertainty Description}

In order to account for the model mismatch, uncertainty in each one of the transfer matrices shown in Fig la should be considered. In the present work we consider, for simplicity, only two types of uncertainty(see Fig 3):

- additive uncertainty, $\Delta_{, m}$, in the transfer function $G_{s m}$,

- additive uncertainty, $\Delta_{s d}$, in the transfer function $G_{s d}$.

Two arguments for selecting this specific uncertainty set are:

-Only uncertainty in $\mathrm{G}_{\text {am }}$ can affect the cloeed-loop stability.

-Since for measurement selection we are comparing the performance obtained using different measurements, the uncertainty associated with the measurements is most important.

Because the uncertainty for the steady state and for the dynamic situations are estimated using different approsches, they will be discussed separately below.

\subsubsection{Steady State Uncertainty Description}

The uncertainty bounds are obtained in the following manner:

-Static gains in the transfer functions matrices, $G_{m m}$ and $G_{s d}$, are measured for a variety of different operating conditions.

-For each element in these matrices, the static gain is bounded by the measured extremes.

-The nominal model is computed by averaging these two extremes.

-A bound on the uncertainty is obtained by computing the difference between the nominal gain and either measured extreme.

Uncertainty in $\mathrm{G}_{\mathrm{s} m}$

The uncertainty in $\mathrm{G}_{m m}$ is modeled as follows:

$$
G_{s m}=\hat{G}_{s m}+\Delta_{s m}^{*}
$$

where

$$
\begin{aligned}
& \hat{G}_{z m}=\left[\begin{array}{ll}
\hat{G}_{T_{z} R} & \hat{G}_{T_{z} H}
\end{array}\right] \\
& \Delta_{s m}^{*}=W_{e_{2}} \Delta_{s m} W_{e_{1}} \\
& W_{e_{1}}=\left(\begin{array}{cc}
W_{r_{s} R} & 0 \\
0 & W_{T_{E} H}
\end{array}\right) \\
& W_{e s}=\left[\begin{array}{ll}
1 & 1
\end{array}\right] \\
& \Delta_{\mathrm{m}}=\left(\begin{array}{cc}
\Delta_{R} & 0 \\
0 & \Delta_{H}
\end{array}\right) \\
& \bar{\sigma}\left(\Delta_{R}\right) \leq 1 \quad \bar{\sigma}\left(\Delta_{H}\right) \leq 1
\end{aligned}
$$

where: R-Recycle flow rate, H-Heating power, $T_{z}$-Temperature measurement used for inference. The gains, $\mathrm{G}_{T_{e} R}$ and $\mathrm{G}_{T_{z} H}$, are identified for different operating points within the selected window of operation (Table 2). These gains are identified by exciting the system with a small step in either of the two manipulated variables while holding the other variable constant. Two sets of experiments are run:
1. changes in heating power holding flow constant - steps from $0-30 \%$ in the heating power for recycle flow rates of 10,12 , 15 , and 17 alpm,

\begin{tabular}{|c|c|c|c|c|c|c|c|}
\hline Therwocouple & $G_{T, 2}$ & $W_{T, R}$ & $\dot{G}_{T, E}$ & $W_{T, H}$ & $G_{\tau_{I} \boldsymbol{T}_{n}}$ & $W_{T_{1} T_{1}}$ & $\begin{array}{c}\text { Correlation } \\
\text { between } \\
\Delta_{R} \text { and } \Delta_{H}\end{array}$ \\
\hline 1 & -2.875 & 0. & 1.2 & $\overline{0.12}$ & 216 & 0.5 & \\
\hline 2 & -3.00 & 0.5 & 1.1 & 0.1 & 2.16 & 0.5 & 0 \\
\hline 3 & -3.08 & 0.08 & 0.96 & 0.08 & 2.35 & $\overline{0.33}$ & 0 \\
\hline 4 & -2.75 & 0.25 & 0.84 & 0.08 & 2.33 & 0.33 & $\overline{0}$ \\
\hline 5 & .2 .58 & 0.06 & 0.7 & 0.06 & 2.5 & 0.195 & 0 \\
\hline 6 & -2.625 & 0.373 & 0.66 & 0.06 & 2.58 & 0.25 & 0 \\
\hline 7 & -2.75 & 0.25 & 0.64 & 0.04 & 2.91 & 0.08 & 0 \\
\hline 8 & $\overline{.2 .75}$ & 0.75 & 0.6 & 0.04 & 2.74 & 0.08 & 0 \\
\hline 9 & -2.75 & 0.75 & 0.56 & 0.04 & 2.74 & 0.08 & 0 \\
\hline 10 & -3.125 & 0.873 & 0.53 & 0.05 & 3.16 & 0.16 & + \\
\hline 11 & .3 .25 & 0.75 & 0.5 & 0.02 & 3.16 & 0.16 & $T$ \\
\hline 12 & -3.375 & 1.120 & 0.4 & 0.04 & 3.33 & 0.16 & $\overline{0}$ \\
\hline$\sqrt{3}$ & -3.125 & 0.875 & 0.53 & 0.04 & 3.5 & 0.16 & $\because$ \\
\hline 14 & .3 .00 & 0.5 & 0.21 & 0.13 & 3.5 & 0.16 & - \\
\hline 15 & .2 .75 & 0.25 & 0.13 & 0.17 & 3.33 & 0.33 & - \\
\hline
\end{tabular}

2. changes in flow rate holding power supplied to heaters constant - steps from 10-12, 12-15, and 15-17 slpm for 15\% power.

The nominal gains, $\hat{G}_{T_{z} R}$ and $\hat{G}_{T_{x} H}$, with their aseciated uncertrinty bounds, $W_{T_{s} R}$ and $W_{T_{s} H}$, are computed as explained above and are presented in Table 2.

Tuble 0.2: A verage teady tate gin with corropooding uncertainty for transfer functions $G_{T_{s},}, G_{T_{s}, A}$, and $G_{T_{s} T_{S}}$

For some choices of thermocouples $\Delta_{R}$ and $\Delta_{H}$ were found to be correlated. For example, it is obeerved that for thermocouples 13,14 , and 15 , for decreasing recycle flow rates, $G_{T_{*} R}$ increases while $\mathrm{G}_{T_{s} H}$ decreases indicating a negative correlation. When this correlation is neglected, the resulting control design will be more conservative when compared with the design for which this correlation is considered.

Uncertainty in $G_{\text {,d }}$

G,d is a single-input single-output transfer function relating the reactor wall temperature to the selected measurement, $\mathbf{T}_{\boldsymbol{x}}$. In this case the uncertainty was measured by imposing steps of $-3^{\circ} \mathrm{C}$ in Dowtherm temperature for 2 different operating points:

$-15 \%$ power, 10 slpm recycle,

$-15 \%$ power, 17 slpm recycle.

With these two experiments the system is excited at the extremes of the selected window of operating conditions. As is done for $G_{m} m$, the steady state gain and the uncertainty asociated with that gain are computed from the results of these experiments. These results are also listed in Table 2.

\subsubsection{Dynamic Uncertainty Description}

The elements of $G_{2 m}$ and $G_{\text {od }}$ are approximated by first order transfer functions with dead time. The values of the time constants and time delays are determined at differeat operating points from the same step response experiments conducted for steady state identification.

Laughlin et al[8] have developed a technique for translating variations in transfer function parameters, i.e. time constants and delays, into an approximately equivalent norm bounded uncertainty representation:

$$
P=\{\tilde{P}(I+W \Delta) ; \sigma(\Delta) \leq 1\}
$$

where $P$ is the "true" uncertain plant, $\tilde{P}$ is the nominal plant and $W$ is the uncertainty weight. Using this method we compute frequency dependent uncertainty weights $W_{\&}$ and $W_{o m}$.

\subsection{Steady State Measurement Selection: Re- sults}

In principle, all possible combinations of temperature measurements should be considered when selecting the measurement set. In the present work we limit the search to a single "best" mear surement. This can be justified by the fact that only one type 
of disturbance is studied in the experiment, i.e., the variation of the reactor wall temperature. As mentioned in Section 2 under ideal conditions a perfect estimator can be designed using a single measurement.

In general, ss more mesourements are used for estimution, more uncertainty is added to the system with a resulting performance degradation. An exception is the case where the transfer functions for different measurements change in a correlated manner in the window of operation (e.g. the changes in $G_{T_{i} A}$ are correleted to the changes in $\mathrm{G}_{T_{j} R}$ ). In this case the performance conld be improved by using more measurements. However, such a correlation was not found in this experiment.

Based on the information in Table 2, we can select the thermo couple to be used in the inferential control scbeme. The weights $W_{i}$ and $W$, used for the computation of $c_{p}^{*}$ are:

$$
W_{d}=4
$$

i.e., the maximal magnitude of the diaturbance considered in the experimeats is $\pm 4^{\circ} \mathrm{C}$ and,

$$
W_{p}=\left[\begin{array}{c|c}
1 & 0 \\
\hline 0 & 0.033
\end{array}\right]
$$

The weight, $W_{p}$, is selected such that concentration errors are of the same order of magnitude as errors in the maximal temperature.

The values of the scalar $c$; for robust performance for each of the measurements in the reactor are computed and shown in Fig 4. The measurements in Fig.4 are numbered beginning from the reactor inlet. It is observed from Fig 4 that $c_{\text {; }}^{*}$ increases as thermocouple \#13 is approached. After this thermocouple, a drastic deterioration in $c$ occurs. This deterioration can be explained by the strong nonlinearity sociated with the formation of the hot spot at the exit of the reactor. As pointed out in the previous section, negative correlation was found between the errors in $\mathrm{G}_{R T_{z}}$ and $\mathrm{G}_{H T_{z}}$ for thermocouple 13,14 and 15 respectively. For comparison $c$; is computed for thermocouple \#13 when the correlation is taken into account $\left(c_{;}^{*}=4.85\right)$ and for the case where the correlation is not considered $\left(c_{*}^{*}=3.5\right)$. This implies that, by neglecting the correlation one overestimates the worst steady state error by $30 \%$.

\subsection{Dynamic Compensator Design}

As explained in Section 2, the dynamic measurement selection is intimately related to the selected dynamic compensator. Thus, the compensator has to be designed before the dynamic measurement selection can be conducted. The transfer matrix of the process, $\mathrm{G}_{\mathrm{cm}}$, is identified from step experiments. It is assumed that $\mathrm{G}_{e m}$ can be modelled as a first order transfer function with time delay. The results of this identification are presented in Table 3

\begin{tabular}{|c|c|c|c|}
\hline & $\boldsymbol{R}$ & $\boldsymbol{H}$ & $T_{\nabla}$ \\
\hline$C_{\bullet}$ & $\frac{0.00011^{-3}}{1-0.012^{-1}}$ & $\frac{-0.0003^{-15}}{1-0.0 \times 1 y^{2}}$ & $\frac{-0.17712^{-2}}{-0.161^{-1}}$ \\
\hline$T_{\text {max }}$ & $\frac{-0.444}{1-0.01312}$ & 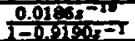 & 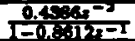 \\
\hline$T_{13}$ & $\frac{-0.547}{1-0.018^{-1}}$ & $\frac{0.01958^{-11}}{1-0.95 .7=1}$ & $\frac{0.552 x^{-2}}{1-0.53 \times 9^{-1}}$ \\
\hline
\end{tabular}
for a sampling time of 40 sec.

Table 3: Transfer function models based on nominal operating conditions

The compensator $Q$ is designed using the MMC factorisation pre sented by Holt and Morari [9].This factorization procedure leads to a dynamically decoupled aystem with the minimal settling time in the controlled variables.

The matrix $G_{a m}$ is factored into an all-pass part and a minimum phase part.

$$
G_{\mathrm{cm}}=G_{\mathrm{cm}}^{\mathrm{A}} G_{\mathrm{cm}}^{\boldsymbol{M}}
$$

The all-pass matrix, $G_{\text {chn }}^{1}$, is a diagonal matrix containing only dead time elements. Using this factorisation,

$$
G_{e m}^{A}=\left[\begin{array}{cc}
z^{-13} & 0 \\
0 & z^{-4}
\end{array}\right]
$$

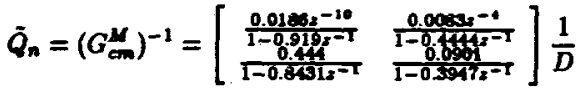

$$
\begin{aligned}
& D=\frac{0.001673 x^{-6}}{\left(1-0.3947 x^{-1}\right)\left(1-0.919 x^{-1}\right)}-\frac{0.00068}{\left(1-0.8431 x^{-1}\right)\left(1-0.4444 z^{-1}\right)}
\end{aligned}
$$

The seros of $D$ are inside the unit circle, and therefore, $\tilde{Q}_{n}$ is stable.

The robustness IMC filter is given by:

$$
F=\frac{\left(1-e^{-T_{0} / \lambda}\right) z}{\left(z-e^{-T_{*} / \lambda}\right)} I
$$

By adjusting $\lambda$ different controllers can be designed with different epeeds of response.

\subsection{Dynamic Measurement Selection:Results}

In order to reduce the total amount of experiments and computation, the dynamic measurement selection is conducted for the thermocouples with the best and second best steady state performance. This corresponds, according to Fig 4 , to thermocouple \#13 $\left(c_{p}^{*}=4.95\right)$ and thermocouple $11\left(c_{p}^{*}=4.14\right)$. The identified bounds for the time constants and time delays for the two selected thermocouples are shown in Table 4. From these bounds, the uncertainty bounds for $G_{e m}$ and $G_{\text {ad }}$ can be computed as explained above. In order to conduct the dynamic measurement selection, all the transfer functions have to be estimated for a nominal operating point and the estimator $E$ has to be construeted. Once again, each one of the transfer functions is modelled as a first order system with time delay. The gain, time delays and time constants for these empirical transfer functions are estimated from step responses for the nominal operating condition (15 slpm recycle flowrate and $15 \%$ heating power). The estimated transfer functions are shown in Table 3. The sampling time for all the experiments is $\mathbf{4 0}$ seconds.

\begin{tabular}{|c|c|c|}
\hline & Time constant(sec) & Delay(sec) \\
\hline$G_{H T_{13}}$ & $661-701$ & $200-600$ \\
\hline$G_{R T_{13}}$ & $164-264$ & 0 \\
\hline$G_{T_{2} T_{13}}$ & $251-300$ & $80-120$ \\
\hline$G_{B T_{11}}$ & $673-1108$ & $250-550$ \\
\hline$G_{R T_{11}}$ & $151-211$ & 0 \\
\hline$G_{T} T_{11}$ & $206-300$ & $50-130$ \\
\hline
\end{tabular}

Table 4:Uncertainty bounds for time constants and delays

Using these models, the estimator is computed. For example, fot thermocouple \#13:

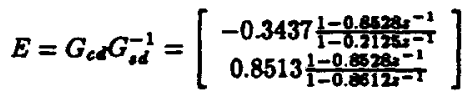

Folbwing the procedure outlined above, robust performance norm bounds are derived for $\bar{\sigma}(F)$ and $\bar{\sigma}(I-F)$ where $F$ is the low pass IMC filter. The bounds $c_{F}$ and $c_{I-F}$ computed for thermocouple \#13 and \#11 as a function of frequency are shown in Fig. $5 \mathrm{a}$ and Fig. $5 \mathrm{~b}$ respectively. According to these figures, thermocouple \#13 is the one with the highest performance bounds . This is due to the fact that the uncertainty in the dynamic parameters is much larger for thermocouple \#11 than for thermocouple \#13 (Table 4). This reinforces the steady state result that indicated thermocouple \#13 as the one with the best steady state performance. Thermocouple \#13 is the sensor cloeest to, but never paseed by, the hot spot in the window of operation.

\subsection{Closed-Loop Experiments}

The main goals of the eloced bop experiments are to tert the sto bility and performance of the inferential contral scheme. By comparing the theoretical predictions of stability and performance to the experimental results, it will be possible to verify the identified uncertainty of the process. All the experiments described below are conducted using thermocouple \#13 for inference. Thus, both 
controlled variables, maximal temperature and exit concentration are inferred from sensor \#13. The stability and the performance results are presented separately in the sequel.

Stability Results It is clear fromig 3 that the stability of the closed loop solely depends on the uncertainty in $\mathrm{G}_{s m}$. Therefore, it is possible to validate the uncertainty $\Delta_{e m}$ by comparing the experimental to the theoretical stability results. The robust stability condition for the $M$ structure shown in Fig $1 \mathrm{~b}$ is given by:

$$
\mu\left(M_{11}\right)=\mu\left(W_{e 1} \tilde{Q_{n}} F E W_{e 2}\right) \leq 1
$$

From this equation it is possible to compute the IMC filter time constant required for stability:

$$
\tau \geq 57 \sec
$$

In order to check this result in the experimental system, we studied the disturbance rejection problem for different operating conditions using different filter time constants: $38 \mathrm{sec}, 57 \mathrm{sec}$ and 140 sec. These experiments are conducted in the following manner:

-The pressure in the Dowtherm container is increased resulting in a slow increase in wall temperature.

-The controller is activated at a predefined operating point.

-The pressure inside Dowtherm container is released causing a step disturbance in wall temperature.

-The manipulated and controlled variables are monitored and stability of the closed-loop determined.

The operating conditions for which the experiments are performed and the stability results are presented in Table 5. As is apparent from experiments $3,4,5$ and 6 in Table 5 , it is more difficult to stabilize the system for lower recycle flow rates. That is, for small recycle flowrates the system appears to be most sensitive to model mismatch. By increasing the filter time constant, we are able to maintain stability at low flow rates, but at the expense of a significantly reduced speed of response (experiment 1).

According to Table 1 the system uncertainty was identified for flow rates between 10 slpm to 18 slpm. However using the filter time constant computed from the robust stability condition (57sec), a limit cycle was obtained for an operating condition corresponding to $12 \mathrm{slpm}$ recycle flowrate and $15 \%$ heating power (experiment 2). Thus, the uncertainty in $G, m$ was underestimated since it does not cover the range 10-12 slpm.

Performance Results The time history of the controlled variables associated with Experiment 5 are presented in Figure 6. Also shown in this figure are the setpoints and the expected deviations for open-loop conditions. It is apparent from these figures that the inferential control scheme rejects the disturbance in wall temperature although it leaves significant steady state offsets in both controlled variables. In the present work, for simplicity, only the steady state performance criterion given by Equation 7 is compared to the experimental results. More specifically, we check for all the experiments

$$
\frac{\left\|W_{p} c(0)\right\|_{2}}{\left\|W_{d}^{-1} d(0)\right\|_{2}} \leq \frac{1}{c_{p}^{*}}
$$

where $c_{p}^{*}=0.21$ for thermocouple \#13. Since the inequality was satisfied for all the performed experiments, we conclude that the estimated steady state uncertainty is not invalidated.

\section{Conclusions}

A robust inferential controller was implemented on an experimental fixed bed methanation reactor. In order to design the inferential controller, a measurement selection technique is applied to determine the thermocouple to be used for inference. Once the measurement is selected, the controller is designed based on IMC theory

The following conclusions can be drawn:

-Due to the inherent system nonlinearities, the inferential controller leaves significant steady state offsets in the controlled variables. These offsets occur even when the system is operated close to the nominal operating condition around which the process was originally identified.

- Correlation between the uncertainty blocks is identified in the experiment. It is important to account for this correlation in the design of the control system. For the reactor, the worst steady state error predicted by the analysis is $30 \%$ larger in the case where the correlation is not considered as compared to the case where this correlation is taken into account.

- From the experiments it is possible to identify regions where the control system is sensitive to model mismatch. In the methanation reactor this occurs at bwer flom rates. It is very important to identify these regions since we are interested in testing the robustness of the feedback system.

-The closed loop experiments can be used to validate the uncertainty estimated for the system. For the reactor it is shown that the robust performance steady state criterion is not violated, indicating that the steady state uncertainty was stimated sufficiently accurately. However this conclusion should be viewed with some caution since complex uncertainty is assumed in the analysis while the steady state uncertainty is real (uncertainty in static gains).

Using the stability results it was shown that the dynamic uncertainty in $\mathrm{G}_{\mathrm{m}}$ is underestimated. The poesible reasons for this underestimation are:

-The system is modelled by assuming first order transfer functions with time delays and is identified from step responses (as opposed to PRBS inputs). As a result the system model may not have been estimated accurately.

-Linear time invariant uncertainty is assumed for the analysis. This is not the best uncertainty description for highly nonlinear systems such as the packed bed reactor. A time varying uncertainty description was applied succesfully in a simulation study by Doyle [10] for the control of a CSTR. In future work we intend to make use of this type of uncertainty description for the packed bed reactor.

Acknowledgment: The authors acknowledge the support of the National Seience Foundation. Hector Budman ackmowledges the support of the Rothschild and Bantrell Foundations.

\section{References}

[1] Webb, C.,H. Budman and M. Morari, "Identifying Prequency Domain Uncertainty Bounds for Robust Controller DesignTheory with application to a Fixed-Bed Reactor", ACC Conf,Pittsburgh,1528-1533,1989.

[2] Lee, J.H. and M. Morari, "Robust Control of NonminimumPhase Systems Through the use of Secondary Measurements: Inferential and Inferential Cascade Control", submitted to Automatica, 1989.

[3] Weber, R. and C. Brosilow, The Use of Secondary Measurements to Improve Control" ,AIChE J.,18:614-623,1972.

[4] Kumar, S. and J.H. Seinfeld, "Optimal Location of Measurements in Tubular Reactors", Chem.Eng.Sci.,33:15071516,1978 .

[5] Harris, T.J.,J.F. MacGregor and J.D. Wright, “An Application of self tuning regulators to catalytic reactor control", In Joint Automat.Contr.Conf.,1978.

[6] Morari M. and E. Zafiriou,Robust Process Control,Prentice Hall,1989.

[7] Hill, C.G.Jr,Chemical Engineering Kineties \& Reactor Design,John Wiley \& Sons, 1977.

[8] Laughlin D.L.,K.G. Jordan and M. Morari, "Internal model control and process uncertainty mapping uncertainty regions for SISO controller design."Int.J.Contr., 44:1675-1698,1986.

[9] Holt, B.R. and M. Morari, "Design of resilient processing plants:The effect of deadtime on dynamic resilience",Chem.Eng.Sci.40:1229-1237,1985.

[10] Doyle, F.J., A. K. Packard and M. Morari, "Robust Coiltroller Design for a Nonlinear CSTR",ACC Conf.,10871093,Pittsburgh,1989. 


\begin{tabular}{|c|c|c|c|c|}
\hline Expit & $\begin{array}{l}\text { Toitial Operatins } \\
\text { Condition: }\end{array}$ & $\begin{array}{l}\text { Robuntoes Filiter } \\
\text { Time Constant }\end{array}$ & $\begin{array}{l}\text { Magnitude of } \\
\text { Disturbance }\end{array}$ & $\begin{array}{l}\text { Subility } \\
\text { Reoult: }\end{array}$ \\
\hline 1 & 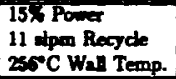 & $140=x$ & $-3+\mathrm{C}$ & Stable \\
\hline 2 & $\begin{array}{l}15 \% \text { Poner } \\
12 \text { dpoe Royyde } \\
250^{\circ} \mathrm{C} \text { Wall Temp. }\end{array}$ & $57=x$ & $-1^{\circ} \mathrm{C}$ & Limit cycle \\
\hline 3 & $\begin{array}{l}\text { 15\% Powe } \\
\text { Il atpon Recycle } \\
256^{\circ} \mathrm{C} \text { Wall Temp. }\end{array}$ & 38 & $-i^{\circ} \mathrm{C}$ & Unatable \\
\hline 4 & $\begin{array}{l}15 \times \text { Poner } \\
13 \text { dpe Recycle } \\
250^{\circ} \mathrm{C} \text { Wal Temp. }\end{array}$ & $38 x$ & $-20 \mathrm{C}$ & Stable \\
\hline 5 & 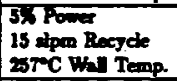 & $38=0$ & $-3^{\circ} \mathrm{C}$ & Stable \\
\hline 6 & $\begin{array}{l}15,17 \text { dpa Rexyde } \\
250^{\circ} \mathrm{C} \text { Wal Temp. }\end{array}$ & $38=x$ & $-{ }^{\circ} \mathrm{C}$ & Stable \\
\hline
\end{tabular}

Thlble 5:Cloned-loop inferential coetrol experiments.

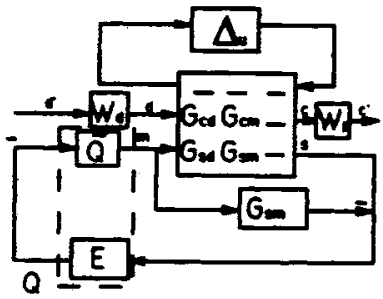

2

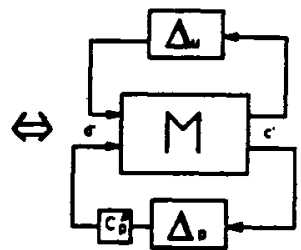

b
Figure 1: Block structure for steady state performance analysis

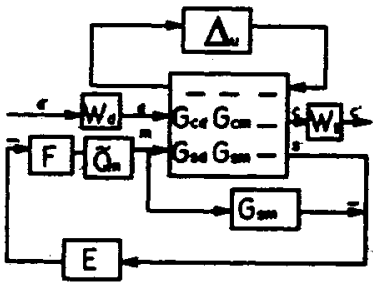

a

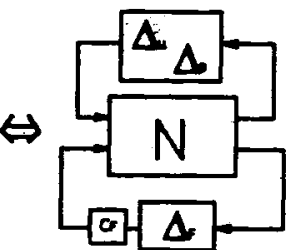

b
Figure 2: Block structure for robust performance analysis

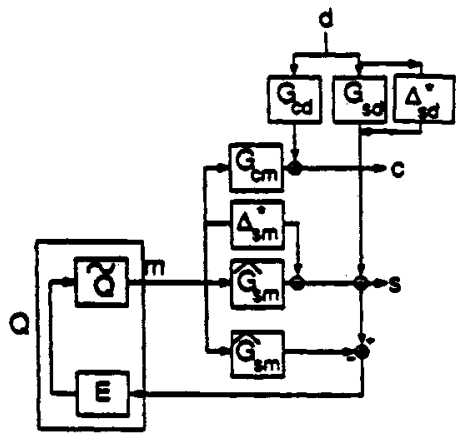

Figure 3: Inferential control applied to an uncertain system

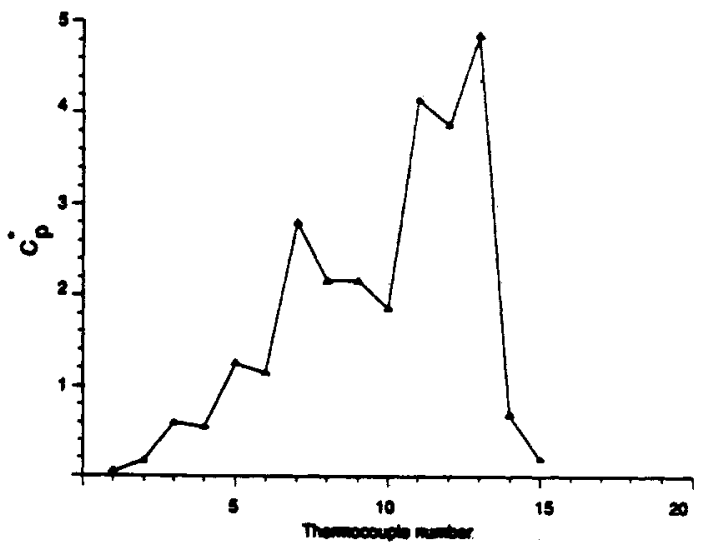

Figure 4: Performance bound $c_{p}^{*}$.
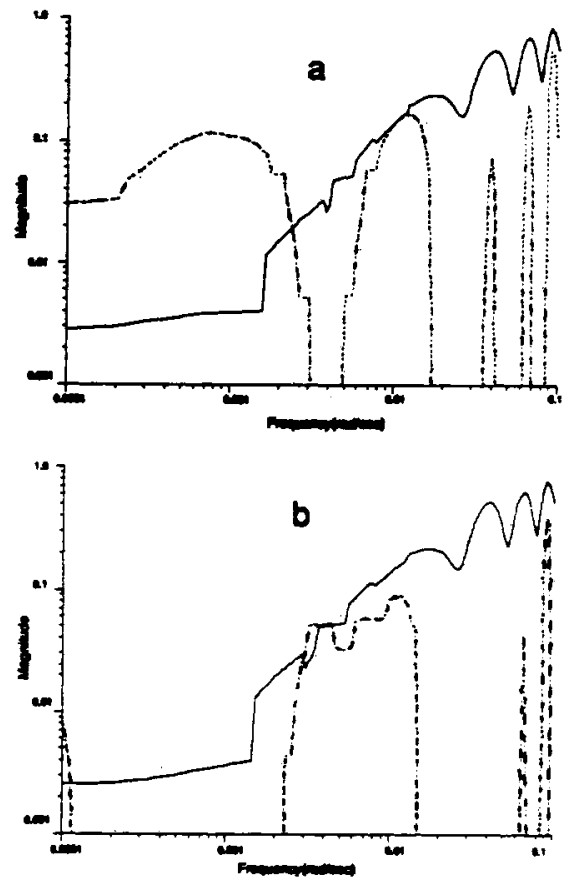

Figure 5: Robust performance bounds for a-thermocouple 13,b-thermocouple 11. (dashed line $c_{I-F}$ solid line $c_{F}$ )
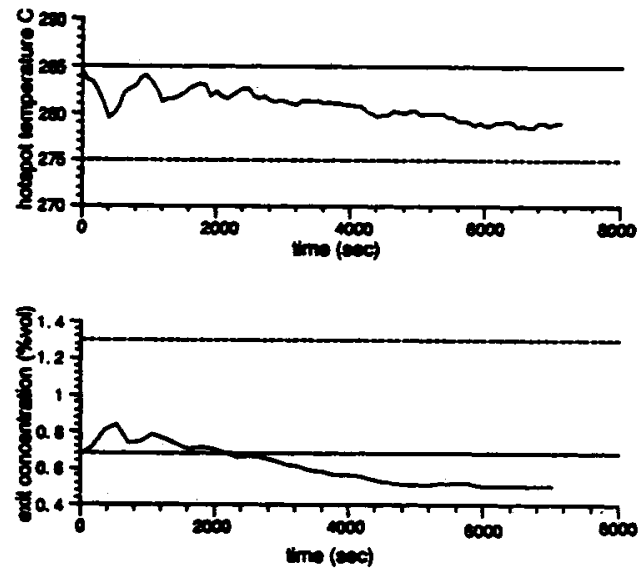

Figure 6: Response of controlled variables to step decrease in wall temperature. (experiment 5).Solid line-set point.Dashed line-expected open loop deviation 